\title{
ON THE UNIFORM ASYMPTOTIC STABILITY OF FUNCTIONAL DIFFERENTIAL EQUATIONS OF THE NEUTRAL TYPE
}

\author{
J. K. HALE ${ }^{1}$ AND A. F. IZE ${ }^{2}$
}

Abstract. Consider the functional equations of neutral type (1) $(d / d t) D\left(t, x_{t}\right)=f\left(t, x_{t}\right)$ and (2) $(d / d t)\left[D\left(t, x_{t}\right)-G\left(t, x_{t}\right)\right]=f\left(t, x_{t}\right)$ $+F\left(t, x_{t}\right)$ where $D, f$ are bounded linear operators from $C[a, b]$ into $R^{n}$ or $C^{n}$ for each fixed $t$ in $[0, \infty), F=F_{1}+F_{2}, G=G_{1}+G_{2},\left|F_{1}(t, \phi)\right|$ $\leqq \nu(t)|\phi|,\left|G_{1}(t, \phi)\right| \leqq \pi(t)|\phi|, \pi(t)$, bounded and for any $\epsilon>0$, there exists $\delta(\epsilon)>0$ such that $\left|F_{2}(t, \phi)\right| \leqq \epsilon|\phi|,\left|G_{2}(t, \phi)\right| \leqq \epsilon|\phi|, t \geqq 0,|\phi|$ $\langle\delta(\epsilon)$. The authors prove that if (1) is uniformly asymptotically stable, then there is a $\zeta_{0}, 0<\zeta_{0}<1$, such that for any $p>0,0<\zeta<\zeta_{0}$, there are constants $\nu_{0}>0, M_{0}>0, s_{0}>0$, such that if $\pi(t)<M_{0}$, $t \geqq s_{0},(1 / p) \int_{t}^{t+p} \nu(s) d s<\zeta \nu_{0}, t>0$, then the solution $x=0$ of $(2)$ is uniformly asymptotically stable. The result generalizes previous results which consider only terms of the form $F_{1}, G_{1}$ or $F_{2}, G_{2}$ but not both simultaneously, and the stronger hypothesis $\lim _{t \rightarrow \infty} \pi(t)=0$.

Suppose $r \geqq 0$ is a given real number, $R=(-\infty, \infty), E$ is a real or complex $n$-dimensional linear vector space with norm $|\cdot|, C([a, b], E)$ is the Banach space of continuous functions mapping the interval $[a, b]$ into $E$ with the topology of uniform convergence. If $[a, b]$ $=[-r, 0]$, we let $C=C([-r, 0], E)$ and designate the norm of an element $\phi$ in $C$ by $|\phi|=\sup _{-r \leq \theta \leq 0}|\phi(\theta)|$. If $\Omega$ is an open subset of $R \times C$ and $f, D: \Omega \rightarrow E$ are given continuous functions, we say that the relation

$$
(d / d t) D\left(t, x_{t}\right)=f\left(t, x_{t}\right)
$$

is a functional differential equation. A function $x$ is said to be a solution of (1) if there are $\sigma \in R, A>0$ such that $x \in C([\sigma-r, \sigma+A), E)$, $\left(t, x_{t}\right) \in \Omega, t \in(\sigma, \sigma+A)$ and $x$ satisfies (1) on $(\sigma, \sigma+A)$. Notice this

Received by the editors June 22, 1970.

AMS 1969 subject classifications. Primary 3475; Secondary 3451.

Key words and phrases. Functional differential equations of neutral type, Banach space, topology of uniform convergence, uniform asymptotic stability, bounded operator, bounded variation, uniformly nonatomic, trajectory, variation of constants formula, uniform stability, exponential asymptotic stability.

1 This research was supported in part by the National Aeronautics and Space Administration under Grant No. NGL 40-002-015 and in part by the Air Force Office of Scientific Research under Grant No. AF-AFOSR 693-67.

2 This research was supported in part by the Conselho Nacional de Pesquisa, in part by Fundação de Amparo á Pesquisa do Estado de São Paulo, and in part by the Air Force Office of Scientific Research under Grant No. AF-AFSOR 693-67B. 
definition implies that $D\left(t, x_{t}\right)$ and not $x(t)$ is continuously differentiable on $(\sigma, \sigma+A)$. For a given $\sigma \in R, \phi \in C,(\sigma, \phi) \in \Omega$, we say $x(\sigma, \phi)$ is a solution of (1) with initial value $(\sigma, \phi)$ if there is an $A>0$ such that $x(\sigma, \phi)$ is a solution of (1) on $[\sigma-r, \sigma+A)$ and $x_{\sigma}(\sigma, \phi)=\phi$.

Our objective is to study the relationship between the uniform asymptotic stability of the linear neutral differential equation

$$
(d / d t) D\left(t, x_{t}\right)=f\left(t, x_{t}\right)
$$

and the perturbed equation

$$
(d / d t)\left[D\left(t, x_{t}\right)-G\left(t, x_{t}\right)\right]=f\left(t, x_{t}\right)+F\left(t, x_{t}\right),
$$

where $D\left(t, x_{t}\right)=\phi(0)-g(t, \phi), g(t, \cdot), f(t, \cdot)$ are bounded linear operators from $C$ in to $E$ for each fixed $t$ in $[0, \infty), g(t, \phi)$ is continuous for $(t, \phi) \in[0, \infty) \times C$,

$$
\begin{aligned}
& g(t, \phi)=\int_{-r}^{0}\left[d_{\theta} \mu(t, \theta)\right] \phi(\theta), \quad f(t, \phi)=\int_{-r}^{0}\left[d_{\theta} \eta(t, \theta)\right]_{\phi}(\theta), \\
& g(t, \phi) \leqq K|\phi|,|f(t, \phi)| \leqq l(t)|\phi|, \quad(t, \phi) \in[0, \infty) \times C,
\end{aligned}
$$

for some nonnegative constant $K$, continuous nonnegative function $l$ and $\mu(t, \cdot), \eta(t, \cdot)$ are $n \times n$ matrix functions of bounded variation on $[-r, 0]$. We also assume that $g$ is uniformly nonatomic at zero, that is, there exists a continuous, nonnegative, nondecreasing function $\gamma(s)$ for $s$ in $[0, r]$ such that $\gamma(0)=0,\left|\int_{-s}^{0}\left[d_{\theta} \mu(t, \theta)\right] \phi(\theta)\right| \leqq \gamma(s)|\phi|$.

Throughout the paper, we assume that $D-G, F$ satisfy enough smoothness conditions to ensure that a solution of (3) exists through each point $(\sigma, \phi) \in[0, \infty) \times C$, is unique, depends continuously upon $(\sigma, \phi)$ and can be continued to the right as long as the trajectory remains in a bounded set in $[0, \infty) \times C$. Sufficient conditions for these properties to be true are contained in [2].

Basic to this investigation is the variation of constants formula given in [1]. If the solution $x_{t}(\sigma, \phi)$ of the linear system is designated by $T(t, \sigma) \phi$, then there is an $n \times n$ matrix function $B(t, s)$ defined for $0 \leqq s \leqq t+r, t \in[0, \infty)$, continuous in $s$ from the right, of bounded variation in $s, B(t, s)=0, t \leqq s \leqq t+r$, such that the solution $x(\sigma, \phi)$ of

(3) is given by

$$
x_{t}(\sigma, \phi)=T(t, \sigma) \phi
$$

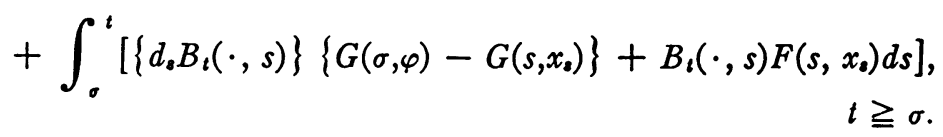


Furthermore, by [1], if the solution $x=0$ of (1) is uniformly asymptotically stable, there are constants $M \geqq 1, \alpha>0$, such that

$$
\begin{aligned}
|T(t, \sigma) \phi| & \leqq M e^{-\alpha(t-\sigma)}|\phi|, & & t \geqq \sigma \geqq 0, \quad \phi \in C, \\
\left|B_{t}(\cdot, s)\right| & \leqq M e^{-\alpha(t-s)}, & & t \geqq s \geqq 0, \\
\int_{\sigma}^{s}\left|d_{u} B_{t}(\cdot, u)\right| & \leqq M e^{-\alpha(t-s)}, & & t \geqq s \geqq \sigma \geqq 0 .
\end{aligned}
$$

In the following we will also assume that

$$
G=G_{1}+G_{2}, \quad F=F_{1}+F_{2},
$$

where

$$
\begin{aligned}
& \left|F_{1}(t, \phi)\right| \leqq \nu(t)|\phi|, \\
& \left|G_{1}(t, \phi)\right| \leqq \pi(t)|\phi|, \quad t \geqq 0, \quad \phi \in C,
\end{aligned}
$$

where $\pi(t), \nu(t)$ are continuous, $\pi(t), \int_{t}^{t+1} \nu(s) d s$ are bounded for $t \geqq 0$ and for any $\epsilon>0$ there is a $\delta(\epsilon)>0$ such that

$$
\left|F_{2}(t, \phi)\right| \leqq \epsilon|\phi|, \quad\left|G_{2}(t, \phi)\right| \leqq \epsilon|\phi|, \quad t \geqq 0,|\phi|<\delta(\epsilon) .
$$

We can now prove the following

Theorem. Suppose $F_{1}, G_{1}$ satisfy (7) and $F_{2}, G_{2}$ satisfy (8). If system (2) is uniformly asymptotically stable, then there is a $\zeta_{0}, 0<\zeta_{0}<1$, such that for any $p>0,0<\zeta<\zeta_{0}$, there are constants $\nu_{0}>0, M_{0}>0, s_{0}>0$, such that if

$$
\begin{aligned}
\pi(t) & <M_{0}, & t \geqq s_{0}, \\
\frac{1}{p} \int_{\imath}^{t+p} \nu(s) d s & \leqq \zeta \nu_{0}, & t \geqq 0,
\end{aligned}
$$

then the solution $x=0$ of (3) is uniformly asymptotically stable.

Proof. Let $R^{+}=[0, \infty)$. The boundedness hypotheses on $\pi(t)$, $\int_{t}^{t+1} \nu(s) d s$ together with an argument similar to the one in Lemma 1, of [3] imply for any $\beta>0$, there are $\delta_{1}(\beta)>0, M_{1}(\beta)>0$ such that for any $\sigma \in R^{+}$the solution $x=x(\sigma, \phi)$ of (3) through $(\sigma, \phi)$ satisfies $\left|x_{t}(\sigma, \phi)\right| \leqq M_{1}(\beta)|\phi|$ for $\sigma \leqq t \leqq \sigma+2 \beta$, provided that $|\phi| \leqq \delta_{1}(\beta)$. From the hypothesis of uniform asymptotic stability there are constants $M \geqq 1, \alpha>0$, such that $B$ and $T$ in (4) satisfy (5). Suppose $0<\zeta<1 /(2 M-1)$. Then $\zeta<1$ and $\zeta<(1+\zeta) / 2 M$. Let $M_{2}(\beta, \zeta)$ $=\max \left[M\left(1+\pi^{*}(0)+\epsilon\right) ; M_{1}(\beta)\right] 2 /(1-\zeta)$. Choose $M_{0}>0, \beta>0, \epsilon>0$ such that 


$$
\begin{aligned}
\stackrel{\operatorname{def}}{=} & M \pi^{*}(0) e^{-\alpha \beta}+M_{2}(\beta, \zeta) M_{0}+M_{2}(\beta, \zeta) \epsilon\left(1+\alpha^{-1}\right)+\zeta \\
& <(1+\zeta) / 2 M,
\end{aligned}
$$

where $\pi^{*}(s)=\sup _{s \leq t} \pi(t)$. The choice of $M_{0}, \beta, \epsilon$ satisfying (11) can be made in the following way. First choose $\beta$ so that

$$
M \pi^{*}(0) e^{-\alpha \beta}<(1+\zeta) / 6 M-\zeta / 3,
$$

then choose $M_{0}$ so that

$$
M_{2}(\beta, \zeta) M_{0}<(1+\zeta) / 6 M-\zeta / 3
$$

and finally choose $\epsilon$ so that

$$
M_{2}(\beta, \zeta) \epsilon\left(1+\alpha^{-1}\right)<(1+\zeta) / 6 M-\zeta / 3 .
$$

Let $s_{0}=\sigma+\beta$ and suppose (9) is satisfied.

From the hypotheses on $F_{2}, G_{2}$, for the above $\epsilon>0$, there is a $\delta_{2}(\epsilon)>0$ such that

$$
\left|F_{2}(t, \phi)\right| \leqq \epsilon|\phi|, \quad\left|G_{2}(t, \phi)\right| \leqq \epsilon|\phi|,
$$

for $|\phi|<\delta_{2}(\epsilon)$. Choose $\delta>0$ such that

$$
M_{2}(\beta, \zeta) \delta<\min \left(\delta_{1}(\beta), \delta_{2}(\epsilon)\right) .
$$

For any $p>0$, choose $\nu_{0}$ so that

$$
p M_{2}(\beta, \zeta) \nu_{0}=\left(e^{\alpha p}-1\right) /\left(2 e^{\alpha p}-1\right)
$$

and suppose (10) is satisfied for this $\nu_{0}$.

If $k=k(t-\sigma)$ is the integer such that $k p \leqq t-\sigma<(k+1) p$ then

$$
\begin{aligned}
\int_{\sigma}^{t} e^{-\alpha(t-u)} \nu(u) d u & =\int_{\sigma+k p}^{t} e^{-\alpha(t-u)} \nu(u) d u+\sum_{j=0}^{k-1} \int_{\sigma+j p}^{\sigma+(j+1) p} e^{-\alpha(t-u)} \nu(u) d u \\
& \leqq p \zeta \nu_{0}+\sum_{j=0}^{k-1} e^{-\alpha(t-\sigma-j p-p)} p \zeta \nu_{0} \\
& =\left[1+e^{-\alpha(t-\sigma-p)} \frac{1-e^{\alpha k p}}{1-e^{\alpha p}}\right] p \zeta \nu_{0} \\
& =\left[1+\frac{e^{\alpha p}}{e^{\alpha p}-1}\left\{e^{-\alpha(t-\sigma-k p)}-e^{-\alpha(t-\sigma)}\right\}\right] p \zeta \nu_{0} \\
& \leqq \frac{2 e^{\alpha p}-1}{e^{\alpha p}-1} p \zeta \nu_{0} \\
& =\frac{\zeta}{M_{2}(\beta, \zeta)}
\end{aligned}
$$


or

$$
M_{2}(\beta, \zeta) \int_{0}^{t} e^{-\alpha(t-u)} \nu(u) d u \leqq \zeta
$$

Furthermore, since $M \leqq(1-\zeta) M_{2}(\beta, \zeta) / 2\left(1+\pi^{*}(0)+\epsilon\right)$, we have

$$
\begin{aligned}
M \int_{0}^{t} e^{-\alpha(t-u)} \nu(u) d u & \leqq \frac{M \zeta}{M_{2}(\beta, \zeta)} \leqq \frac{(1-\zeta) \zeta}{2\left(1+\pi^{*}(0)+\epsilon\right)} \\
& \leqq \frac{(1-\zeta) \zeta}{2}<\zeta .
\end{aligned}
$$

Let us write the variation of constants formula for the solution $x=x(\sigma, \phi)$ of (3) in the form

$$
\begin{aligned}
x_{t}= & T(t, \sigma) \phi+\left(\int_{0}^{*}+\int_{0}^{t}\right)\left[d_{u} B_{t}(\cdot, u)\right]\left[G_{1}(\sigma, \phi)-G_{1}\left(u, x_{u}\right)\right] \\
& +\int_{0}^{t} B_{t}(\cdot, u) F_{1}\left(u, x_{u}\right) d u \\
& +\int_{0}^{t}\left[d_{u} B_{t}(\cdot, u)\right]\left[G_{2}(\sigma, \phi)-G_{2}\left(u, x_{u}\right)\right] \\
& +\int_{0}^{t} B_{\iota}(\cdot, u) F_{2}\left(u, x_{u}\right) d u
\end{aligned}
$$

for $\sigma \leqq s \leqq t$.

Therefore, as long as $\left|x_{t}\right| \leqq \delta_{2}(\epsilon)$, it follows from (5) and the hypotheses on $F, G$, that

$$
\begin{aligned}
& \left|x_{\imath}\right| \leqq M\left(1+\pi^{*}(0)+\epsilon\right)|\phi| e^{-\alpha(t-\sigma)} \\
& +M\left[\pi^{*}(\sigma) e^{-\alpha(t-s)}+\pi^{*}(s)+\epsilon\left(1+\alpha^{-1}\right)\right. \\
& \left.+\int_{0}^{t} e^{-\alpha(t-u)} \nu(u) d u\right] \sup _{\sigma \leqq u \leqq}\left|x_{u}\right|
\end{aligned}
$$

for $\sigma \leqq s \leqq t$. If we take $s=s_{0}=\sigma+\beta$ and use our estimates on $\beta, \epsilon, M_{0}$ and (9), (13), (11), then

$$
\begin{aligned}
\left|x_{t}\right| & \leqq M\left(1+\pi^{*}(0)+\epsilon\right)|\phi|+\eta \sup _{\sigma \leqq u \leqq t}\left|x_{u}\right| \\
& \leqq \frac{1-\zeta}{2} M_{2}|\phi|+\eta \sup _{\sigma \leqq u \leqq t}\left|x_{u}\right|
\end{aligned}
$$


for $t \geqq \sigma+2 \beta$ as long as $\left|x_{t}\right| \leqq \delta_{2}(\epsilon)$. If $\delta$ is chosen as above and $|\phi|<\delta$, then we know that

$$
\left|x_{t}\right| \leqq M_{1}(\beta)|\phi| \leqq(1-\zeta) M_{2}(\beta, \epsilon)|\phi| / 2 \leqq M_{2}(\beta, \epsilon)|\phi| \leqq \delta_{2}(\epsilon)
$$

for $\sigma \leqq t \leqq \sigma+2 \beta$. Therefore,

$$
\left|x_{t}\right| \leqq \frac{1-\zeta}{2} M_{2}(\beta, \epsilon)|\phi|+\eta \sup _{\sigma \leqq u \leqq t}\left|x_{u}\right|
$$

for all $t \geqq \sigma$ for which $\left|x_{t}\right| \leqq \delta_{2}(\epsilon)$. Consequently, for $\left|x_{t}\right| \leqq \delta_{2}(\epsilon)$,

$$
\begin{aligned}
\sup _{\sigma \leqq u \leqq t}\left|x_{u}\right| & \leqq \frac{1-\zeta}{2(1-\eta)} M_{2}(\beta, \epsilon)|\phi| \\
& \leqq \frac{M(1-\zeta)}{2 M-1-\zeta} M_{2}(\beta, \epsilon)|\phi| \\
& \leqq M_{2}(\beta, \epsilon)|\phi| \leqq \delta_{2}(\epsilon)
\end{aligned}
$$

for $|\phi|<\delta$ since $M \geqq 1$. The continuation theorem implies that $x(t)$ is defined for $t \geqq \sigma-r,(15)$ is satisfied for $t \geqq \sigma$ and the solution $x=0$ of (3) is uniformly stable.

For $s=s_{0}=\sigma+\beta$ in (14) and $t \geqq \sigma+2 \beta$, it follows from (14) and the estimates (15) and (13) that

$$
\begin{aligned}
\left|x_{t}\right| \leqq M\left\{\left(1+\pi^{*}(0)+\epsilon\right) e^{-\alpha(t-\sigma)}+\pi^{*}(\sigma) M e^{-\alpha\left(t-s_{0}\right)}\right. \\
\left.\quad+\pi^{*}\left(s_{0}\right) M_{2}(\beta, \zeta)+M_{2}(\beta, \zeta) \epsilon\left(1+\alpha^{-1}\right)+\zeta\right\}|\phi| \\
\\
\leqq M\left\{\left(1+\pi^{*}(0)+\epsilon\right) e^{-\alpha(t-\sigma)}+\eta\right\}|\phi| \\
\leqq M\left\{\left(1+\pi^{*}(0)+\epsilon\right) e^{-\alpha(t-\sigma)}+(1+\zeta) / 2 M\right\}|\phi| .
\end{aligned}
$$

For any $\delta_{0},(1+\zeta) / 2<\delta_{0}<1$, choose $T \geqq 2 \beta$ so large that

$$
M e^{-\alpha T}\left(1+\pi^{*}(0)+\epsilon\right)+(1+\zeta) / 2<\delta_{0} .
$$

For $t \geqq \sigma+T$, it follows that

$$
\left|x_{i}\right| \leqq \delta_{0}|\phi| \text {. }
$$

Since $T$ is independent of $\sigma$ and $\phi$, this clearly implies exponential asymptotic stability and proves the theorem.

In [1], asymptotic stability theorems of the above type were proved for systems which contained either terms of the form $F_{1}, G_{1}$ or $F_{2}, G_{2}$ but not both simultaneously. In addition to combining these results into one, the more significant part of the above theorem is the fact that uniform asymptotic stability is proved under the weak hypothesis (9). In [1], it was assumed that $\pi(t) \rightarrow 0$ as $t \rightarrow \infty$. 


\section{REFERENCES}

1. J. K. Hale and M. A. Cruz, Asymptotic behavior of neutral functional differential equations, Arch. Rational Mech. Anal. 34 (1969), 331-353.

2. - Existence, uniqueness and continuous dependence for hereditary systems, Ann. Mat. Pura Appl. (4) 85 (1970), 63-82.

3. J. K. Hale and K. R. Meyer, A class of functional equations of neutral type, Mem. Amer. Math. Soc. No. 76 (1967). MR 36 \#6889.

Brown University, Providence, Rhode Island 02912

Universidade de São Paulo, São Carlos, São Paulo, Brasil 